\title{
胶体与界面化学
}

郝京诚

山东大学, 胶体与界面化学教育部重点实验室, 济南 250100

\section{Colloid and Interface Chemistry}

\section{Jingcheng Hao}

Key Laboratory of Colloid and Interface Chemistry (Ministry of Education), Shandong University, Jinan 250100, P. R. China. Email: jhao@sdu.edu.cn.

Published online: May 11, 2020.

胶体与界面化学是研究分散体系的物理化 学性质和界面现象的科学 1,2 。该学科与实际结合 紧密, 并与其他学科密切相关, 其基本原理在物理 化学的各分支学科中最具基础性、理论性和应用 性。近年来, 我国学者在胶体分散体系与界面化学 领域的研究中取得了突破性的进展, 很多研究结 果得到了国际同行的关注和认可, 为提升我国在 该领域的国际学术地位起到了重大作用。然而, 由 于国内学者更加关注纳米材料、能源、生物医药等 领域的前沿研究, 相较于国际上在胶体与界面化 学领域的研究, 国内的研究方向也有所偏移。当 然, 纳米材料化学和超分子化学等新兴交叉领域 的渗入, 以及胶体分散体系现代表征技术的快速 发展和理论化学的进步, 对胶体与界面化学的基 础和应用研究都起到了助推作用。因此, 对我国近 年来在该领域的工作进行介绍、梳理、归纳, 有助 于研究者了解我国的研究现状, 选择适合自己的 研究方向和内容。

本专辑收集了我国部分胶体与界面化学领 域的学者在胶体分散体系和两亲分子组装体系物 理化学性质、结构、性能与应用方面的研究成果, 以及对相关研究的综述介绍。胶体分散体系研究 方面, 展示了黄建滨、房喻、齐利民、Zhan Chen、 To Ngai、杨恒权、冯玉军团队的新概念、新思路 和应用等方面的研究结果; 在两亲分子组装体系 方面, 展示了王毅琳、间学海、韩杰、郭荣、董金 风、李学丰、刘鸣华团队的创新理论、实验合成等 方面的研究结果。衰心希望《物理化学学报》的广 大读者群, 特别是胶体与界面化学的读者群, 能够
喜欢这些文章, 并从中得到收获和启迪。

\section{I 胶体分散体系的综述与创新研究成果}

相界面广泛存在, 是胶体分散体系的重要内 容。黄建滨团队 ${ }^{3}$ 从相对广义的 “两亲分子” 概念 出发, 提出了 “油 $-\mathrm{CO}_{2}$ 两亲分子” 概念, 在该概念 基础的指导下, 设计合成了一系列多酯头基为亲 酯基团的 “油- $\mathrm{CO}_{2}$ 两亲分子”, 探究了超临界二 氧化碳 $\left(\mathrm{scCO}_{2}\right)$ 与白油混相体系中的助混规律与效 果。新颖薄膜材料, 例如 LB膜的构建及应用, 已 成为物理化学学科发展的热点。房喻团队 ${ }^{4}$ 长期致 力于苂光敏感薄膜的创制、薄膜器件化和薄膜器 件阵列的研究工作, 发展了系列荧光敏感薄膜材 料, 研制了针对爆炸物、毒品、挥发性有机污染物 等有害物质的高性能荧光传感器和探测设备, 实 现了技术转化和规模生产。结合近期研究进展, 他 们围绕了薄膜基菼光气体传感器的检测/监测应 用, 讨论了传感器创制中涂层化学的科学问题, 综 述了相关研究前景和发展所面临的挑战。环糊精 是一类典型的超分子主体，具有亲水的表面和不 同尺寸的疏水内腔, 在水溶液中与疏水的小分子 容易发生自组装。齐利民团队 5 利用主客体组装的 策略, 通过 $\alpha$-环糊精 $(\alpha-C D)$ 与金纳米棒表面修饰的 十六烷基三甲基溴化铵 (CTAB)的超分子主客体作 用, 在水溶液中成功实现了金纳米棒的头对头和 肩并肩可控组装体材料的制备, 为非球形纳米基 元的可控自组装提供了新思路。仿生防污高分子 涂层的构建可用于解决海洋生物附着于船舶对设 施造成危害, 并可降低燃料消耗, 已经被界面化学 
研究领域关注, 成为新的热点内容。美国密歇根大 学Zhan Chen研究团队6通过使用和频(SFG)振动光 谱技术实现了对防污材料的界面分析工作。该文 中Chen等人总结了两性离子高分子、混合电荷式 高分子和两性类肽高分子等防污材料, 论述了这 些涂层材料的界面性质以及盐离子对界面水分子 的作用, 同时也介绍了防污材料与蛋白质及海藻 之间的作用。构建具有响应性的组装结构, 特别是 以刺激响应性表面活性剂聚集形成不同聚集结构 以实现其响应功能, 已成为表面活性剂科学的热 点内容。颗粒稳定的胶体分散体系, 特别是 Pickering乳液, 近年来得到了越来越多的关注, 但 胶体颗粒对界面的稳定作用和胶体颗粒之间相互 作用的机制仍需要深入探索。香港中文大学魏涛 团队 7 在胶体颗粒稳定的界面领域开展了深入系统 的研究。该文中作者系统阐述了胶体颗粒稳定的 乳液和泡沫的研究现状, 回顾了相关研究发展的 历史，同时介绍了胶体颗粒稳定的分散体系在包 埋、食品、控释和干水制备等领域的应用。在关于 Pickering乳液的应用研究中, 杨恒权团队 8 发展了 一种溶剂诱导的Pickering乳液可逆翻转策略, 实 现了酶的原位分离和高效循环利用, 这是该团队 近年来关于Pickering乳液应用的新进展。液体弹 珠是颗粒稳定的胶体分散体系, 粉末颗粒包裹形 成的不润湿性液滴, 近年来得到胶体与界面学者 的广泛关注。冯玉军团队 9 针对近十年来液体弹珠 的研究进展, 综述了液体弹珠的制备策略、形成机 理、物理性质以及相关应用, 尤其是在微反应器领 域的应用, 展望了液体弹珠的发展方向与应用前 景。

\section{II 两亲分子组装体系的综述与创新研究成果}

表面活性剂在精细化学品工业和技术领域中 具有广泛的应用价值, 是胶体与界面化学研究的 核心与基础。随着合成化学技术的发展, 新颖结构 的表面活性剂不断出现。通过联接基将 2 个或 2 个 以上的传统表面活性剂分子在亲水基或接近亲水 基处连接在一起的Gemini表面活性剂, 由于其特 殊的结构和优异的表面化学性能引起了研究者的 强烈兴趣。王毅琳团队 ${ }^{10}$ 合成了一系列疏水链或联 接基团上含有酰胺基或酯基的可生物降解阳离子 季铵盐型Gemini表面活性剂, 同时合成了酰胺基 的单链表面活性剂以及疏水链和联接基团上同时 含有酰胺键的Gemini表面活性剂。通过表面张力、 电导、苂光、等温滴定量热和 ${ }^{1} \mathrm{H}$ NMR波谱测定, 系统研究了降解基团结构和位置对 Gemini表面活
性剂聚集行为的影响, 以及 Gemini表面活性剂和 单体表面活性剂在表面活性和聚集行为方面的差 异, 给出了合成Gemini表面活性剂的重要物理化 学性质参数。肽分子具有氨基酸规整排列、高度稳 定的一级结构, 其结构明确、可编辑、生物安全性 高, 肽分子间具有氢键等非共价键相互作用, 以上 特点构成了肽分子在超分子组装中的优势。近年 来, 间学海团队 ${ }^{11}$ 在肽分子的超分子组装体系的结 构和功能构建方面取得了突破性进展。他们综述 了肽基超分子胶体研究领域所面临的关键性科学 问题, 对这类材料在能源光催化、疾病光诊疗领域 的应用做了重点论述。表面活性剂蠕虫状胶束和 小分子水凝胶具有独特的聚集体结构和流变学特 性, 其中表面活性剂蠕虫状胶束已经在油田开采 中作为清洁压裂液被广泛应用, 而小分子水凝胶 具有三维网络结构, 该结构在变形和压力下难以 改变。韩杰和郭荣团队12 研究了非离子表面活性剂 Brij 30与离子液体表面活性剂 $\mathrm{C}_{16} \mathrm{imC}_{8} \mathrm{Br}$ 的模型体 系, 通过流变性能测定和冷冻刻蚀透射电子显微 镜的结构表征, 证明了蠕虫状胶束向凝胶的转变, 并以Zeta电位和核磁共振化学位移探究了两者之 间的相互作用。董金凤和李学丰团队 ${ }^{13}$ 长期开展响 应性表面活性剂聚集结构和性能的研究, 他们利 用十六烷基三甲基氢氧化铵和不同结构的甲氧基 取代的肉桂酸衍生物构建了光响应蠕虫状胶束体 系, 研究了甲氧取代基的位置和数量对蠕虫状胶 束结构与光响应性的影响, 明确了光诱导蠕虫状 胶束结构转变过程中甲氧基取代位置的重要作 用。刘鸣华团队 ${ }^{14}$ 将具有良好光学性质的喹喔啉衍 生物修饰于 $\mathrm{L}-$ 谷氨酸基两亲分子中, 得到了含有 较大 $\pi$-共轭头基的手性功能化凝胶因子, 凝胶因 子可以自组装形成由纳米纤维组成的三维网络凝 胶, 该凝胶材料具有多重外界刺激响应性, 为超分 子手性光学开关的应用提供了一条新途径。

\section{References}

(1) Zhao, G. X.; Zhu, B. Y. Action Principle of Surfactants; China Light Industry Press: Beijing; 2003. [赵国玺, 朱㻉瑶. 表面活性剂作用原 理. 北京: 中国轻工业出版社, 2003.]

(2) Liu, H. G.; Sun, D. J.; Hao, J. C. New Colloids and Interface Chemistry; Chemical Industry Press: Beijing; 2016. [刘洪国, 孙德 军, 郝京诚. 新编胶体与界面化学. 北京: 化学工业出版社, 2016.]

(3) Liao, P.; Liu, Z.; Liu, K.; Ma, C.; Zhu, Z.; Yang, S.; Lü, W.; Yang, Y.; Huang, J. Acta Phys. -Chim. Sin. 2020, 36, 1907034. [廖培龙, 刘泽宇, 刘卡尔顿, 马骋, 朱志扬, 杨思玉, 吕文峰, 杨永智, 
黄建滨. 物理化学学报, 2020, 36, 1907034.]

doi: 10.3866/PKU.WHXB201907034

(4) Liu, T.; Miao, R.; Peng, H.; Liu, J.; Ding, L.; Fang, Y. Acta

Phys. -Chim. Sin. 2020, 36, 1908025. [刘太宏, 苗荣, 彭浩南, 刘静, 丁立平, 房喻. 物理化学学报, 2020, 36, 1908025.]

doi: 10.3866/PKU.WHXB201908025

(5) Xiao, J.; Qi, L. Acta Phys. -Chim. Sin. 2020, 36, 1910001. [肖军燕, 齐利民. 物理化学学报, 2020, 36, 1910001.]

doi: 10.3866/PKU.WHXB201910001

(6) Zhang, C.; Crisci, R.; Chen, Z. Acta Phys. -Chim. Sin. 2020, 36, 1910003. [Chengcheng Zhang, Ralph Crisci, Zhan Chen. 物理化学 学报, 2020,36, 1910003.] doi: 10.3866/PKU.WHXB201910003

(7) Sun, G.; Yi, Z.; Ngai, T. Acta Phys. -Chim. Sin. 2020, 36, 1910005. [孙冠卿, 易宗霖, 魏涛. 物理化学学报, 2020, 36, 1910005.] doi: 10.3866/PKU.WHXB201910005

(8) Zou, H.; Ettelaie, R.; Yan, S.; Xue, N.; Yang, H. Acta Phys. -Chim. Sin. 2020, 36, 1910006. [邹厚兵, Rammile Ettelaie, 间帅, 薛楠, 杨恒权. 物理化学学报, 2020, 36, 1910006.] doi: 10.3866/PKU.WHXB201910006

(9) Luo, X.; Zhang, X.; Feng, Y. Acta Phys. -Chim. Sin. 2020, 36,
1910007. [罗新杰, 张熙, 冯玉军. 物理化学学报, 2020, 36, 1910007.] doi: 10.3866/PKU.WHXB201910007

(10) Wang, Y.; Deng, M.; Tang, Y.; Han, Y.; Huang, X.; Hou, Y.; Wang, Y. Acta Phys. -Chim. Sin. 2020, 36, 1909046. [王英雄, 邓曼丽, 唐永强, 韩玉淳, 黄旭, 侯研博, 王毅琳. 物理化学学报, 2020, 36, 1909046.] doi: 10.3866/PKU.WHXB201909046

(11) Xing, L.; Zuo, Q.; Yan, X. Acta Phys. -Chim. Sin. 2020, 36, 1909048. [邢荵荵, 邹千里, 问学海. 物理化学学报, 2020, 36, 1909048.] doi: 10.3866/PKU.WHXB201909048

(12) Hu, Y.; Han, J.; Guo, R. Acta Phys. -Chim. Sin. 2020, 36, 1909049. [胡益民, 韩杰, 郭荣. 物理化学学报, 2020, 36, 1909049.] doi: 10.3866/PKU.WHXB201909049

(13) Liu, T.; Xue, F.; Yi, P.; Xia, Z.; Dong, J.; Li, X. Acta Phys. -Chim. Sin. 2020, 36, 1910004. [刘同庆，薛芳芳，易萍，夏志宇，董金凤， 李学丰. 物理化学学报, 2020, 36, 1910004.] doi: 10.3866/PKU.WHXB201910004

(14) Wang, H.; Xu, L.; Liu, M. Acta Phys. -Chim. Sin. 2020, 36, 1910036. [王含笑, 徐俪菲, 刘鸣华. 物理化学学报, 2020, 36, 1910036.] doi: 10.3866/PKU.WHXB201910036 